\title{
Fibroblast growth factor 11 (FGF11) promotes non-small cell lung cancer (NSCLC) progression by regulating hypoxia signaling pathway
}

Xiaowei $\mathrm{Wu}^{1+}{ }^{+}$, Minjie $\mathrm{Li}^{2+}$, Ying $\mathrm{Li}^{3 \dagger}$, Yu Deng ${ }^{1}$, Shun Ke${ }^{4}$, Fan $\mathrm{Li}^{1}$, Yujin Wang ${ }^{5}$ and Shuchang Zhou ${ }^{{ }^{*}}$ (1)

\begin{abstract}
Background: Accumulating evidence highlights the critical roles of fibroblast growth factors (FGFs) in regulating the progression of multiple human cancers, including non-small cell lung cancer (NSCLC). In this study, we investigated the role of FGF11 in the progression of NSCLC.

Methods: Previously published transcriptomic data (GSE75037 and GSE81089) were used to compare FGF11 expression level between NSCLC tumor tissues and adjacent normal tissues. 100 cases of NSCLC tumor tissues and 30 cases of matched adjacent normal tissues were used to validate FGF11 expression at mRNA and protein level by qPCR and immunohistochemistry. Bioinformatics analysis and dual luciferase reporter analysis were performed to confirm the regulatory effect of miR-525-5p on FGF11 expression. CCK-8 assay and transwell migration assay were employed to examine cellular proliferation, migration and invasion. Gene set enrichment analysis (GSEA) was performed to identify the signaling pathway associated with FGF11 expression. Finally, the functional role of FGF11 in NSCLC tumor growth was evaluated by in vivo study.
\end{abstract}

Results: FGF11 was upregulated in NSCLC tumor tissues and tumor cell lines. High FGF11 expression was associated with a poor prognosis in NSCLC patients. In vitro loss- and gain-of function experiments demonstrated that FGF11 knockdown inhibited, whereas FGF11 overexpression promoted the proliferation, migration and invasion of NSCLC cells. Dual luciferase reporter assay confirmed that FGF11 was downregulated by miR-525-5p, and the effect of FGF11 on cell proliferation, migration and invasion could be interfered by miR-525-5p. GSEA analysis further revealed that FGF11 expression was enriched with genes in hypoxia signaling pathway and the oncogenic function of FGF11 could be suppressed by knocking down HIF-1 a in NSCLC cells. Moreover, FGF11 knockdown suppressed NSCLC tumor growth whereas FGF11 overexpression promoted tumor growth in vivo.

Conclusions: Our study showed that FGF11 functions as an oncogene in tumor NSCLC progression. miR-525-5p seems to negatively regulate FGF11 and the oncogenic role of FGF11 is dependent on the upregulation of HIF-1a. Our study suggests that targeting FGF11 and HIF-1 a may serve as novel strategies for the treatment of NSCLC.

\footnotetext{
*Correspondence: ferhk336@163.com

${ }^{\dagger}$ Xiaowei Wu, Minjie Li and Ying Li contributed equally to this work

${ }^{5}$ Department of Radiology, Tongji Hospital, Tongji Medical Collage

of Huazhong University of Science and Technology, 430030, Wuhan, Hubei, China

Full list of author information is available at the end of the article
}

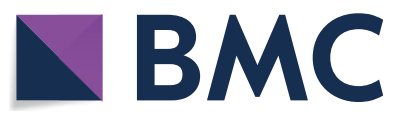

The Author(s) 2021. Open Access This article is licensed under a Creative Commons Attribution 4.0 International License, which permits use, sharing, adaptation, distribution and reproduction in any medium or format, as long as you give appropriate credit to the original author(s) and the source, provide a link to the Creative Commons licence, and indicate if changes were made. The images or other third party material in this article are included in the article's Creative Commons licence, unless indicated otherwise in a credit line to the material. If material is not included in the article's Creative Commons licence and your intended use is not permitted by statutory regulation or exceeds the permitted use, you will need to obtain permission directly from the copyright holder. To view a copy of this licence, visit http://creativecommons.org/licenses/by/4.0/. The Creative Commons Public Domain Dedication waiver (http://creativeco mmons.org/publicdomain/zero/1.0/) applies to the data made available in this article, unless otherwise stated in a credit line to the data. 
Keywords: NSCLC, FGF11, HIF-1a, Hypoxia, Cell proliferation

\section{Background}

According to the Global Cancer Observatory (GLOBOCAN), the world is facing an increasing incidence and mortality of cancer, and lung cancer is the most prevalent type of cancer causing the mortality [1]. Lung cancer is usually subtyped into small cell lung cancer (SCLC) and non-small cell lung cancer (NSCLC), which account for approximately $20 \%$ and $80 \%$ of diagnosed cases of lung cancer respectively $[2,3]$. Despite the advancement of diagnosis and treatment, the management of NSCLC remains one of the most serious medical challenges [4, 5]. Understanding the factors and mechanisms underlying the progression can provide insights into the development of novel therapeutic strategies targeting NSCLC.

Fibroblast growth factor (FGF) family members play important roles in a variety of tumorigenesis by activating multiple signaling pathways to support cell proliferation and promote epithelial-mesenchymal transition (EMT) [6-8]. Previous report has revealed that downregulating fibroblast growth factor 5 can inhibit the proliferation and invasion of NSCLC cells [9]. As one important member of FGF family, the implication of FGF11 and the relevant signaling processes remain to be investigated in NSCLC $[10,11]$.

microRNAs (miRNAs) are small non-coding RNAs which function in regulating mRNA transcription and post-transcriptional stability $[12,13]$. The dysregulation of miRNAs has been implicated in a wide spectrum of human diseases especially in the progression of different types of cancers, including gastric cancer [14], breast cancer [15], lung cancer [16] and colorectal cancer [17].

At present, the prognostic role of FGF11 was only reported in rare cancers, such as nasopharyngeal carcinoma [18] and prostate cancer [19]. Its roles in other physiological processes such as bone resorption and endothelial tube formation have also been reported [20, 21]. A previous study showed that copy number variations (CNVs) of FGF11 gene was correlated with the risk of lung cancer in heavy smokers [22], suggesting a potential role of FGF11 in lung cancer initiation. Hypoxia induced factors-1 alpha (HIF-1 $\alpha$ ) plays a key role in hypoxia signaling pathways which is dysregulated lung cancer [23-26]. FGF11 could be induced by hypoxia, which in turn promotes the function of HIF-1 $\alpha$ in a positive feedback loop in endothelial cells [21]. However, the functional role of FGF11-HIF- $1 \alpha$ axis in cancer development remains unclear.

Through the analysis of previously published transcriptomic data, we found that FGF11 was upregulated in NSCLC tumor tissues, which was also associated with poor prognosis of the patients. Based on target prediction using multiple miRNA databases, we found that miR-525-5p could target and suppress FGF11 expression, suggesting that miR-525-5p is a negative regulator of FGF11. GSEA analysis further revealed that in lung tumor tissues, a high expression of FGF11 was enriched with genes in hypoxia signaling pathway. Functional experiment provided evidence that the oncogenic function of FGF11 could be suppressed by knocking down HIF- $1 \alpha$ in NSCLC cells. In summary, our study indicates that FGF11 is an oncogenic factor in NSCLC. We identified miR-525-5p and HIF- $1 \alpha$ as the negative and positive regulator in FGF11-dependent function, suggesting that targeting these molecules can serve as novel therapeutic approaches for treating NSCLC.

\section{Material and methods \\ Patients and clinical follow up}

100 cases of NSCLC tumor tissues and 30 cases of matched adjacent normal lung tissues were obtained from patients who were newly diagnosed with NSCLC by histopathological analysis. The samples were collected by surgery at Tongji Hospital. All the patients did not receive any other treatment before surgical operation. After surgery, all the samples were snap-frozen in liquid nitrogen. The use of human samples was approved by the Ethics Committee of Tongji Hospital, and all patients and/or their relatives have signed the informed consents. To monitor the survival condition, all the patients were clinically followed for four years. Patients who failed to return for clinical observation or died of accidents were excluded from survival analysis. The clinical characteristics of the 100 NSCLC patients (Histology, Stage, etc.) were shown in Additional file 1: Table S1. Patients were classified into FGF11 high and FGF11 low group $(\mathrm{n}=50$ in each group) based on the median of FGF11 level quantified by qPCR analysis in their NSCLC samples. The median expression level is 6.40 (FGF11 normalized to GAPDH expression).

\section{Cell culture}

Four human NSCLC cell lines (A549, NCI-H460, CALU3, H1975), and human normal lung epithelial cell line (BEAS-2B) were purchased from ATCC (Manassas, VA, USA). All cells were cultured in RPMI 1640 medium (Gibco, USA) which supplemented with $10 \%$ FBS (Gibco, USA), $100 \mathrm{U} / \mathrm{ml}$ penicillin and $100 \mathrm{mg} / \mathrm{ml}$ streptomycin at $37{ }^{\circ} \mathrm{C}$ in a humidified incubator with $5 \% \mathrm{CO}_{2}$. 


\section{RNA extraction and qPCR analysis}

Total RNA was purified from NSCLC tumor cell lines $\left(\sim 5 \times 10^{\wedge 5}\right.$ cells $)$ and tissues $(1 \sim 2 \mathrm{~g})$ using RNeasy mini kit (Qiagen, USA). $5 \mu \mathrm{g}$ purified total RNA was reverse transcribed into cDNA using the PrimeScript RT reagent kit (Cat\# RR037A, Takara, China). miRNA was transcribed using the miRNA specific RT primers which were designed and synthesized by Guangzhou RiboBio, Co., Ltd.

Quantitative real-time PCR (qPCR) reactions were performed on ABI Prism 7500 real time PCR instrument (Applied Biosystems, CA, USA) using the SYBR Green PCR Master Mix (Takara). The relative expression of target gene was determined by $2^{-\Delta \Delta C t}$ method. GAPDH and U6 was selected as the internal control genes for normalizing mRNA and miRNA expression respectively. Primer sequences of FGF11, HIF- $1 \alpha$ and GAPDH, U6 and miR-525-5p were listed as following: FGF11 forward: 5'-GGCATCGTCACCAAACTG TT-3'; reverse: 5'-GCAGTCCCTCAGCATTCATG-3'; HIF- $1 \alpha$ forward: $5^{\prime}$-GACAGCCTCACCAAACAG AG-3'; reverse: 5'-GTAGCTGCATGATCGTCTGG -3'; GAPDH forward: 5'-CTGACTTCAACAGCGACA CC-3'; reverse: 5'-CTGACTTCAACAGCGACACC-3'; U6 forward: 5'-GCTTCGAGGCAGGTTACATG-3'; reverse: 5'-GCAACACACAACATCTCCCA-3'. miR525-5p forward: $5^{\prime}$-GCGGTCCCTCTCCAAATGT-3' reverse: 5'-AGTGCAGGGTCCGAGGTATT-3'.

\section{shRNA knockdown, siRNA knockdown and FGF11 overexpression}

pLKO.1-Puro lentiviral vector was used for shRNAmediated gene silencing, and pLenti-puro plasmid was used for FGF11 overexpression. Lentiviral plasmids overexpressing FGF11 (containing cDNA of FGF11) or carrying FGF11-shRNA and sh-Negative Control (shNC) were constructed by GenePharma Co. Ltd. (Shanghai, China). The packaging of recombinant lentivirus was performed in $293 \mathrm{~T}$ cells by GenePharma Co. Ltd. (Shanghai, China). To generate stable shRNA-mediated knockdown or FGF11 overexpression, $1 \times 10^{5}$ cells were seeded in a 24-well plate. When cells reached at $50 \sim 60 \%$ confluence, cells were infected with recombinant lentivirus at a MOI (multiplicity of infection) $=5$, in the presence of $10 \mu \mathrm{g}$ polybrene (Sigma, tr-1003-g). Infected cells were selected with $1.0 \mu \mathrm{g} / \mathrm{mL}$ puromycin for two weeks to eliminate the uninfected cells before further experiment. qPCR and western blot were performed to confirm the efficiency of shRNA-mediated knockdown and FGF11 overexpression.
miR-525-5p mimic and miR-NC were purchased from Guangzhou RiboBio (Guangzhou, China): miR-525-5p mimic: 5'-CUCCAGAGGGAUGCACUUUCU-3'.

miR-NC: $5^{\prime}$-UUCUCCGAACGUGUCACGU-3'. Control siRNA and HIF- $\alpha$ siRNA (sc-39464) were purchased from Santa Cruz Biotechnology (Texa, USA). The miRNA mimic/inhibitor and siRNAs were transfected into cells using Lipofectamine 3000 (Invitrogen, L3000001) according to the manufacturer's instructions. $50 \mathrm{nM}$ of each molecule was used for transfection and functional experiments were performed $48 \mathrm{~h}$ post-transfection.

\section{Dual luciferase reporter assay}

To demonstrate the functional interaction between FGF11 and miR-525-5p, FGF11 3'UTR region containing miR-525-5p binding sites (named as WT) and fragment containing site-directed mutagenesis (named as MUT) were cloned into psiCHECK-2.0 vector. The reporter plasmid and Renilla luciferase (hRlucneo) control plasmid were co-transfected into $293 \mathrm{~T}$ cells with either MiR$525-5 \mathrm{p}$ mimic or inhibitor in a 24 -well plate $\left(2 \times 10^{\wedge} 5\right.$ cells/well) using Lipofectamine 3000 reagent according to the manufacturer's instructions (Invitrogen, L3000001). $48 \mathrm{~h}$ post transfection, the relative luciferase activities were measured using Dual-Luciferase Reporter Assay Kit (Promega, E1910) on a luminescence microplate reader (Infinite 200 PRO; Tecan). The relative firefly luciferase activity in the reporter plasmid was normalized to that of Renilla luciferase (hRlucneo) control plasmid.

\section{Cell Counting Kit 8 (CCK-8) assay}

Cell Counting Kit-8 (CCK-8; Dojindo, Japan) assay was used to examine the cell proliferation capacity. NSCLC cells were seeded in a 96-well plate at a density of 3,000 cells/well, then cultured for indicated time period. $10 \mu \mathrm{l}$ of CCK- 8 solution was added to the cell culture at each time point, the plates were incubated for $3 \mathrm{~h}$. $10 \mu \mathrm{L}$ CCK-8 reagent was added to each well at indicated time point and incubated in the incubator for $1 \mathrm{~h}$. A microplate reader (Bio-Rad, CA, USA) was used to detect the absorbance value (OD value) in each well at $450 \mathrm{~nm}$.

\section{Colony formation assay}

Colony formation assay was used to evaluate the longterm proliferation potential. Cells were infected with recombinant lentivirus carrying shRNA or co-transfected with corresponding microRNA mimics, inhibitor or siRNA, as mentioned in the shRNA and siRNA expression Sect. $48 \mathrm{~h}$ post-transfection, cells were seeded into a 6-well plate at the density of 200 cells/well after cell counting. The culture medium was changed every two days. After two weeks' culture, cells were fixed with paraformaldehyde and stained using Giemsa Stain Kit 
(Abcam, ab150670) according to the manufacturer's instructions. Finally, the number of colonies formed in each condition was counted using Leica AM6000 microscope.

\section{Cell migration and invasion assay}

Cell migration ability was determined by wound healing assay and cell invasion assay. For wound healing assay, cells were seeded into a 6 -well plate of $2 \times 10^{5}$ cells. When the confluency reached at $70-80 \%$, a scratch was created on cell monolayer using a sterile $100 \mu \mathrm{l}$ tip. Cells were cultured for another $48 \mathrm{~h}$ and the closure of the scratch was imaged using a phase contrast microscope (Leica AM6000 microscope.).

For the invasion assay, Matrigel (354,230, BD, USA) was diluted in an appropriate proportion and coated on the bottom of transwell chamber (CLS3398, Sigma, Germany). Cells were trypsinized and then resuspended in serum-free medium. After cell counting, $1 \times 10^{4}$ cells were seeded into the upper chamber in medium with $3 \%$ FBS, and $500 \mu \mathrm{L}$ medium with $10 \%$ FBS was added into the lower chamber. After incubator for $48 \mathrm{~h}$, cells were fixed with $4 \%$ paraformaldehyde for $10 \mathrm{~min}$ at room temperature and staining with $0.5 \%$ crystal violet solution (c0755, Sigma, Germany) in 25\% methanol for $20 \mathrm{~min}$. The invading and migrating cells were image and counted using Leica AM6000 microscope.

\section{Apoptosis assay by flow cytometry}

Apoptosis assay was performed using the PE-Annexin V Apoptosis Detection Kit I (BD biosciences, 559763). After lentiviral infection or transfection with microRNA mimics, cells were washed twice with pre-cold PBS. Cells were harvested by trypsinization and collected by centrifuging at $500 \mathrm{xg}$ for $5 \mathrm{~min}$. The cell pellet was resuspended in $1 \mathrm{ml}$ medium with $5 \mu \mathrm{l}$ of PE-Annexin $\mathrm{V}$ and $5 \mu \mathrm{l}$ of 7-AAD solution for $15 \mathrm{~min}$ at room temperature. Stained cells were analyzed using Attune NxT Flow Cytometer (BD, Biosciences, USA). Collected data were analyzed by Flow Jo software (Version 13.0, BD).

\section{Western blot analysis}

Total protein was extracted from NSCLC tumor tissues and cells using RIPA lysis buffer containing protease inhibitor cocktail (Invitrogen, USA), then quantified by a BCA Protein assay kit (Solarbio, Beijing, China). 10 ug protein was used for SDS-PAGE electrophoresis. Separated protein in SDS_PAGE gel was transferred onto the PVDF membrane (BioRed, USA). After blocking with 5\% skimmed milk for $1 \mathrm{~h}$, the membrane was then incubated with primary antibodies: FGF11 (1:1000; Cell Signaling Technologies \#3139, MA, USA), HIF-1 $\alpha$ (1:1000, Cell Signaling Technologies \#3716), $\beta$-Actin (13E5) (1:2000,
Cell Signaling Technologies \#4970) and GAPDH (1:2000; Cell Signaling Technologies \#2118) for $2 \mathrm{~h}$ or overnight at $4^{\circ} \mathrm{C}$. The membrane was washed 3 times with TBST for 5 min each. After wash, the membrane was further incubated with HRP-linked secondary antibody (1:3000; Cell signaling \#7074, MA, USA) at room temperature for $1 \mathrm{~h}$. Then the membrane was washed 4 times with $1 \times$ TBST and the protein bands were visualized using an enhanced chemiluminescence kit (Santa Cruz, TX, USA) and photographed on a gel imager system (Bio-Rad).

\section{Animal tumor xenograft model}

$\mathrm{Balb} / \mathrm{c}$ nude mice (female, aged 6-8 weeks, weight 18-20 g) were used to perform the in vivo animal experiment. Mice were purchased from the Slac Laboratory Animal Center (Shanghai, China) and maintained under pathogen-free conditions. $5 \times 10^{5} \mathrm{~A} 549$ and/or NCIH460 cells stably infected with FGF11, FGF11 shRNA or sh-NC lentivirus were resuspended in $100 \mu \mathrm{l}$ of PBS and subcutaneously injected into the right flank of mice (5 mice in each group). The tumors were monitored using a caliper weekly and the tumor volume was calculated using the formula: V(tumor) $=0.5 \times$ length $\times$ width

${ }^{2}$. Seven weeks after tumor cell inoculation, all the mice were euthanized by $\mathrm{CO}_{2}$ asphyxiation, and the xenograft tumors were excised and weighted. All experimental procedures of the use of animal were approved by the Ethics Committee of Tongji Hospital. All the efforts were made to minimize animal suffering according to the NIH Guide for the Care and Use of Laboratory Animals.

\section{IHC and H\&E staining}

Immunohistochemical staining (IHC) of Ki-67 protein or FGF11 was performed on 4-mm sections of formalin-fixed paraffin-embedded (FFPE) tumor tissue using VENTANA BenchMark Special Stain platform (Roche, Indianapolis, IN, USA) based on the manufacturer's instructions. Antibody used for Ki-67 IHC staining was Anti-Ki67 antibody (ab15580, $0.5 \mu \mathrm{g} / \mathrm{ml}$ in TBST, Abcam, USA). Antibody used for FGF11 IHC staining was AntiFGF11 antibody [MM0282-6J20] (ab89713, $0.5 \mu \mathrm{g} / \mathrm{ml}$ in TBST, Abcam, USA).

Hematoxylin and Eosin (H\&E) staining was performed using H\&E Stain Kit (ab245880, Abcam, USA). Deparaffinized/hydrated section was incubated in adequate Hematoxylin solution for $5 \mathrm{~min}$. The section was rinsed twice with distilled water to remove excess stain. Then adequate Bluing Reagent was applied to completely cover tissue section and incubate for 30 secs. After washing with distilled water, the section was dehydrated in absolute alcohol, followed by staining with Eosin Y Solution to completely cover tissue for $2-3 \mathrm{~min}$. The section 
(See figure on next page.)

Fig. 1 FGF11 is upregulated in human NSCLC tissues and is associated with poor prognosis. A The relative expression of FGF11 in GSE75037 dataset. B The relative expression of FGF11 in GSE81089 database. C qPCR analysis of FGF11 expression in 100 cases of NSCLC tissues and 30 cases of adjacent normal tissues. D The examination of FGF11 protein level by immunohistochemistry (IHC) in NSCLC tumor tissue and adjacent normal tissue. E Kaplan-Meier plotter survival analysis of 1144 cases of NSCLC patients from TCGA database. F Kaplan-Meier plotter analysis of survival probability of FGF11 high expression group $(n=50)$ and the low expression group $(n=50)$ in NSCLC patients. G qPCR analysis of FGF11 expression in NSCLC cell lines (A549, NCI-H460, CALU3, H1975) and human normal lung epithelial cell line (BEAS-2B). H Western bot analysis of FGF11 protein level in NSCLC cell lines (A549, NCI-H460, CALU3, H1975) and human normal lung epithelial cell line (BEAS-2B). The above data in (G and H) are the summary of the measurements of 3 independent experiments (mean \pm standard deviation). ${ }^{*} P<0.05,{ }^{* *} P<0.01,{ }^{* *} P<0.001$

was rinsed using absolute alcohol for three times and mounted in synthetic resin for observation.

\section{Bioinformatic analysis}

The gene expression data of NSCLC patients were retrieved from Gene Expression Omnibus (GEO) repository (GSE75037 and GSE81089). Gene Set Enrichment Analysis (GSEA) was performed according to the instructions on Molecular Signatures Database (MSigDB): https://www.gsea-msigdb.org/gsea/index.jsp. The expression characteristics of FGF11 and the prognosis of NSCLC patients were extracted from TCGA database through the website tool GEPIA (http://gepia. cancer-pku.cn/index.html). MicroRNA target prediction databases were used for predicting FGF11 binding microRNAs: Starbase (http://starbase.sysu.edu.cn/index.php); miRBD (http://mirdb.org/) and Targetscan (http://www. targetscan.org/vert_72/).

\section{Statistical analysis}

All results were analyzed by GraphPad Prism 8.0 software (GraphPad Software, San Diego, CA, USA). Statistical analyses were performed using Student's $t$-test, one-way analysis of variance (0ne-way ANOVA), Kaplan Meier plotter analysis or $\mathrm{X}^{2}$ test. Correlation between FGF11 and HIF-1 $\alpha$ expression in NSCLC tissues was assessed by Pearson's correlation analysis. $P<0.05$ was considered as significant statistical difference.

\section{Results}

FGF11 is upregulated in human NSCLC tissues and significantly correlated with the prognosis

We first analyzed the previously published transcriptome data (GSE75037 and GSE81089) containing NSCLC tumor sample and the adjacent normal tissues. We found that the expression level of FGF11 was significantly higher in NSCLC tumor tissues than that of normal tissues (Fig. 1A and B). To further validate this observation, we collected 100 NSCLC tumor tissues and 30 matched adjacent normal lung tissues. qPCR analysis revealed that FGF11 expression level was much higher in NSCLC tissues when compared to the normal tissues (Fig. 1C). Moreover, immunohistochemistry (IHC) analysis confirmed a higher protein level of FGF11 NSCLC tumor tissue (Fig. 1D).

To further evaluate the clinical significance of FGF11 on patient survival, we first selected 1144 cases of NSCLC patients from TCGA database, and allocated them into a FGF11-high expression group $(n=571$; $>$ median expression level of all cases) and a FGF11-low expression group ( $\mathrm{n}=573 ;<$ median expression level of all cases). Kaplan Meier plotter analysis showed that high FGF11 level was associated with an overall survival of in NSCLC patients (Fig. 1E). Meanwhile, the survival analysis of NSCLC cases we collected showed a similar tendency (Fig. 1F). We further performed qPCR and western blot analysis using NSCLC cell lines and a human normal lung epithelial cell line (BEAS-2B). mRNA and protein levels were significantly higher in all NSCLC cell lines when compared to normal lung epithelial cell line (Fig. 1G and H). Collectively, those data suggest that FGF11 expression level correlates with the prognosis in NSCLC patients.

\section{FGF11 knockdown inhibits NSCLC cell proliferation and promotes apoptosis}

The elevated level of FGF11 in human NSCLC samples prompted us to further explore the biological functions of FGF11 in NSCLC cells. FGF11 was silenced in A549 and NCI-H460 cells by infection with FGF11 shRNA (shFGF11) lentivirus. The knockdown efficiency was confirmed by qPCR analysis (Fig. 2A) and western blot (Fig. 2B). CCK-8 proliferation assay was used to examine the cell proliferation capacity after FGF11 silencing in A549 and NCI-H460 cells. The knockdown of FGF11 notably suppressed the cell proliferation in both cell lines (Fig. 2C). The knockdown of FGF11 also significantly inhibited the colony formation ability of NSCLC (Fig. 2D). In addition, apoptosis assay by flow cytometry revealed an increased percentage of apoptotic cells when FGF11 was knocked down (Fig. 2E). The above data demonstrate an indispensable role of FGF11 in supporting NSCLC proliferation.

\section{FGF11 overexpression promotes NSCLC cell proliferation} To further support a role of FGF11 in promoting NSCLC cell proliferation, we overexpressed FGF11 in A549 and 


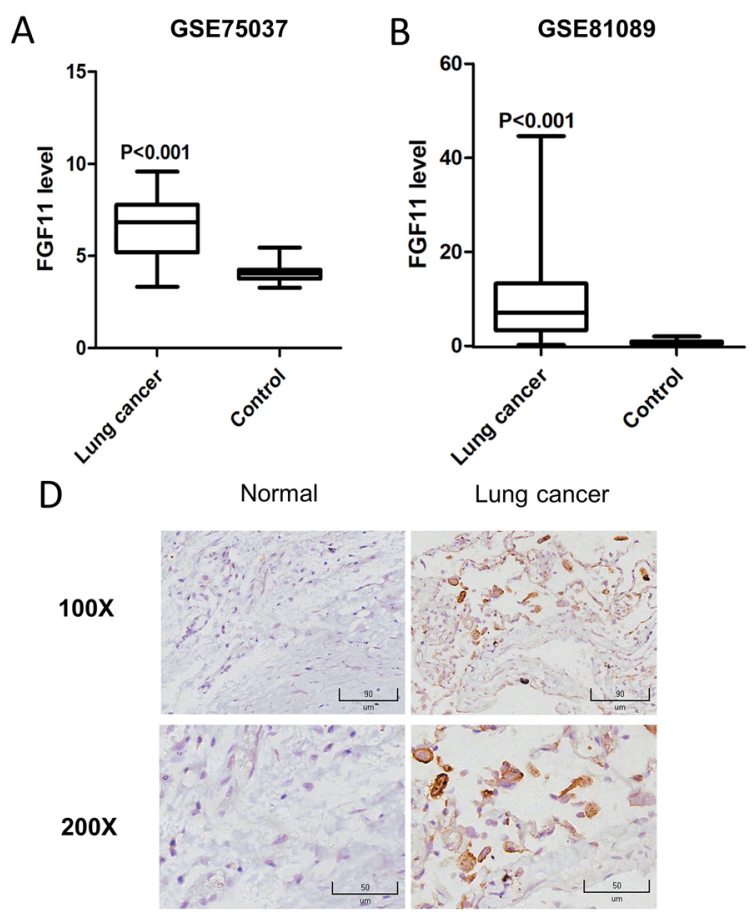

\section{C}
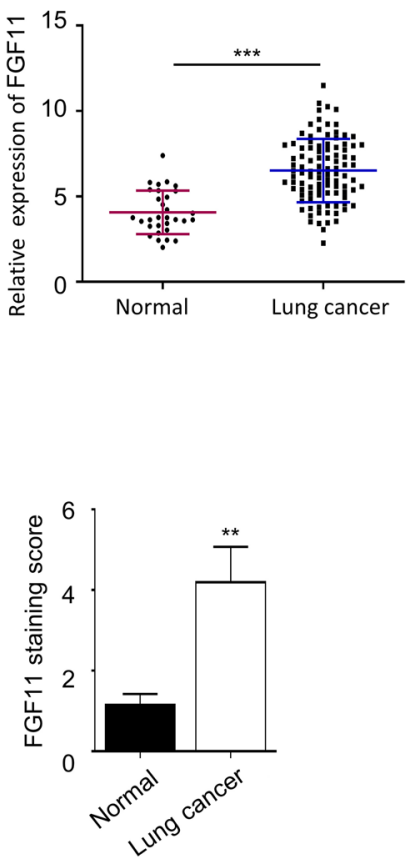

E

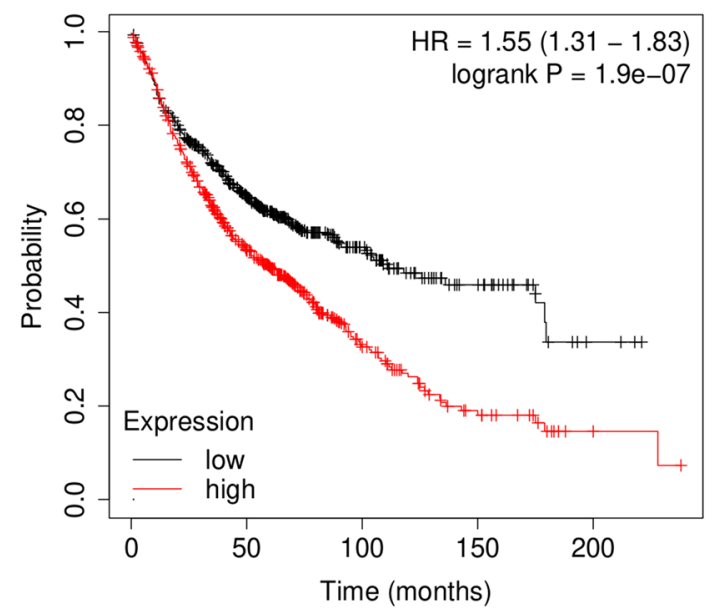

$\mathrm{F}$
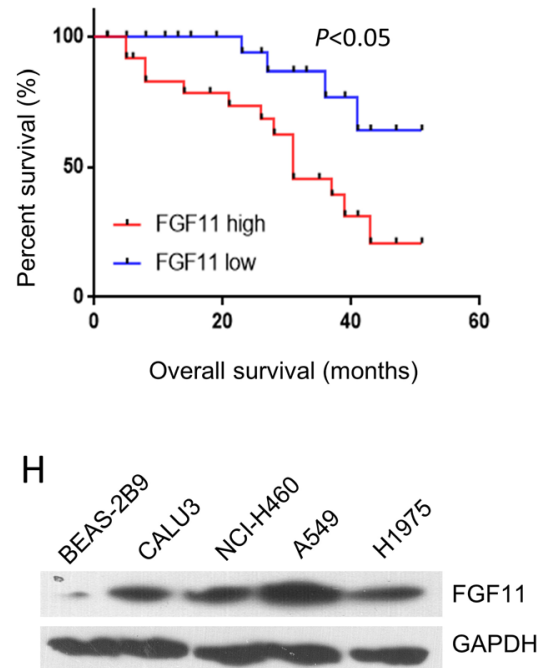

G
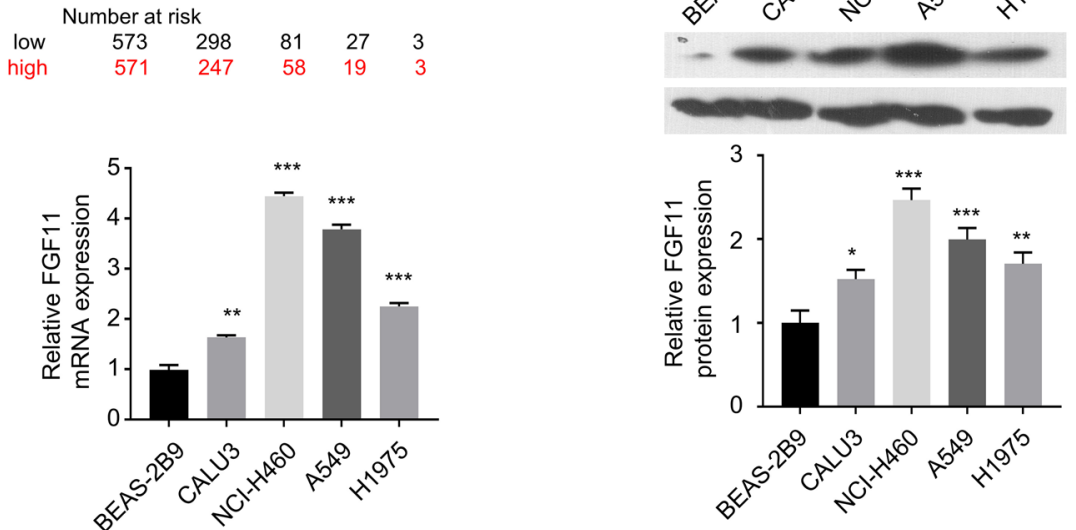

Fig. 1 (See legend on previous page.) 
A
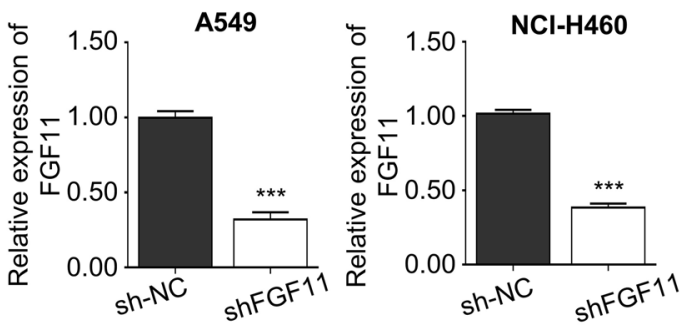

C
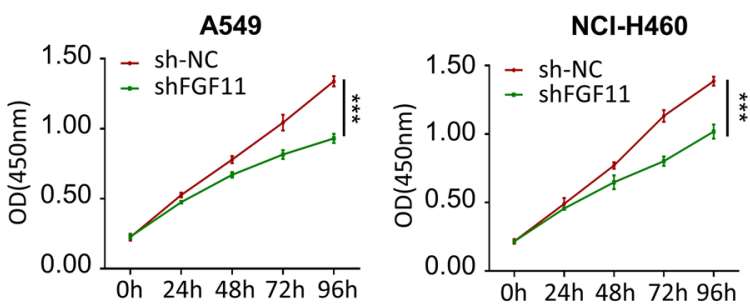

D
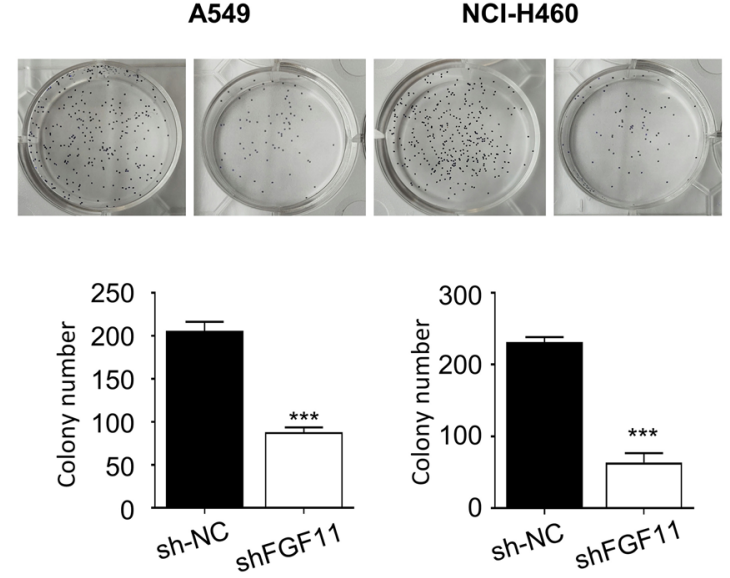

B

A549

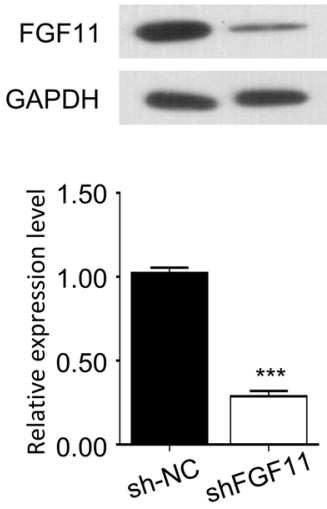

FGF11

GAPDH
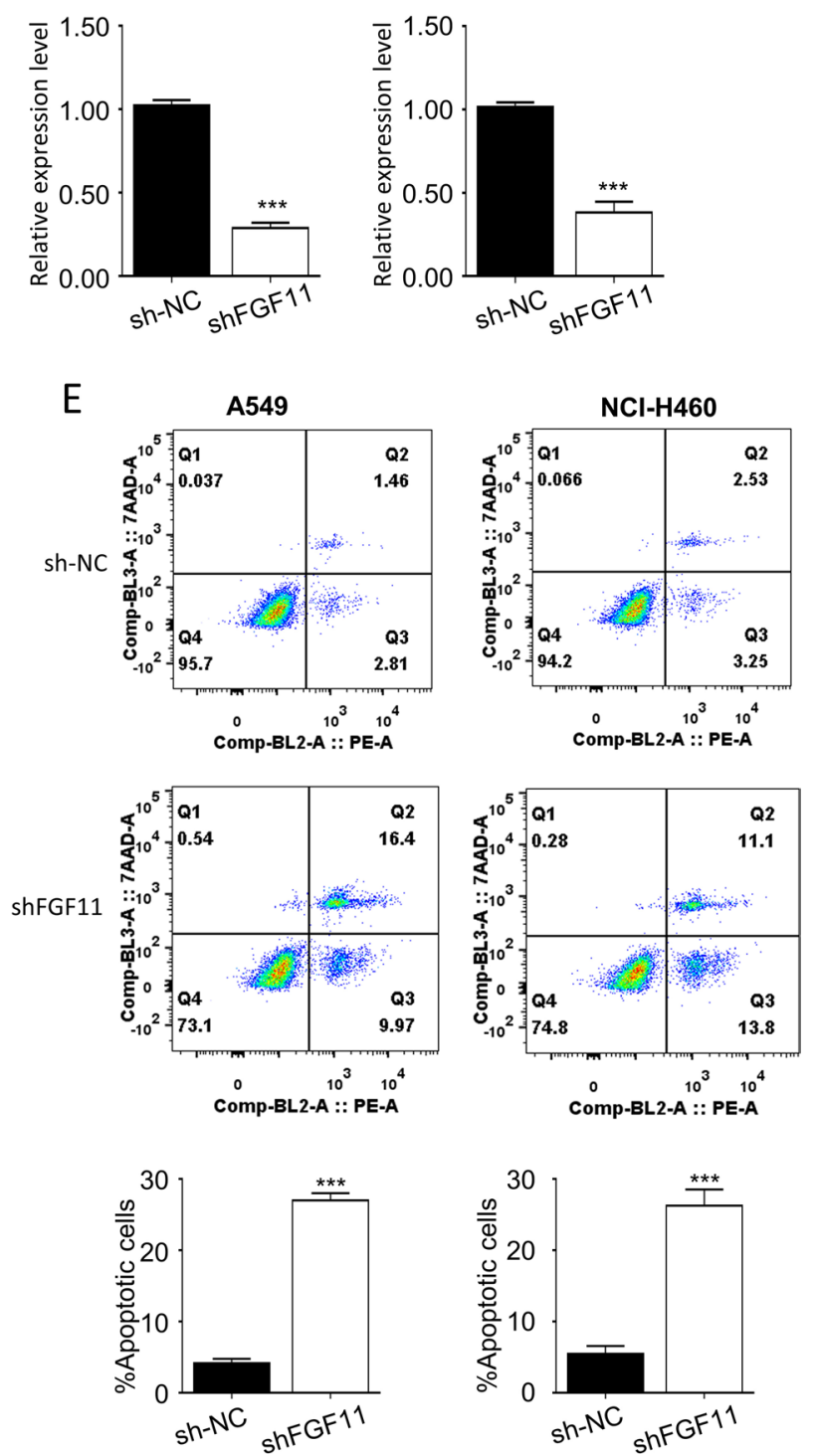

Fig. 2 Knockdown of FGF11 inhibits NSCLC cell proliferation and promotes cell apoptosis. Cells were infected with lentivirus carrying FGF11 shRNA (shFGF11) or control shRNA (sh-NC). A qPCR analysis of FGF11 mRNA expression in A549 and NCl-H460 cells after shRNA introduction. B Western blot analysis of FGF11 protein levels in A549 and NCl-H460 cells. C The proliferation of A549 and NCl-H460 cells after shRNA introduction was detected by CCK-8 assay. D Colony formation assay of A549 and NCI-H460 cells after shRNA introduction. E Flow cytometry analysis of cells stained with Annexin-V and 7-AAD. Data are the summary of the measurements of 3 independent experiments (mean \pm standard deviation). ${ }^{*}<<0.05$, ${ }^{* *}$ $\mathrm{P}<0.01,{ }^{* * *} \mathrm{P}<0.005$ vs. sh-NC or empty vector-infected cells

NCI-H460 cells by infection with lentivirus carrying FGF11 cDNA clone. Lentivirus-mediated overexpression significantly increased the level of FGF11 by about 3 times (Fig. 3A, and B). CCK-8 proliferation assay demonstrated that FGF11 overexpression promoted the tumor cell proliferation as well as the ability in colony formation (Fig. 3C and D), supporting an oncogenic role of FGF11 in NSCLC cells. 


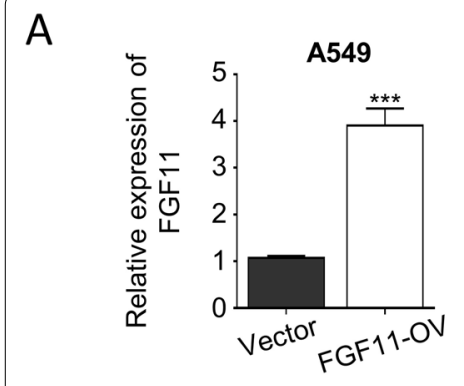

B
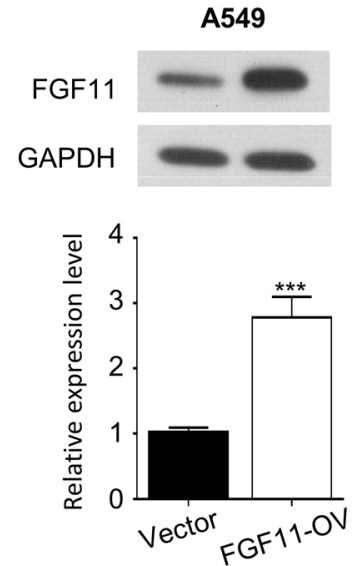
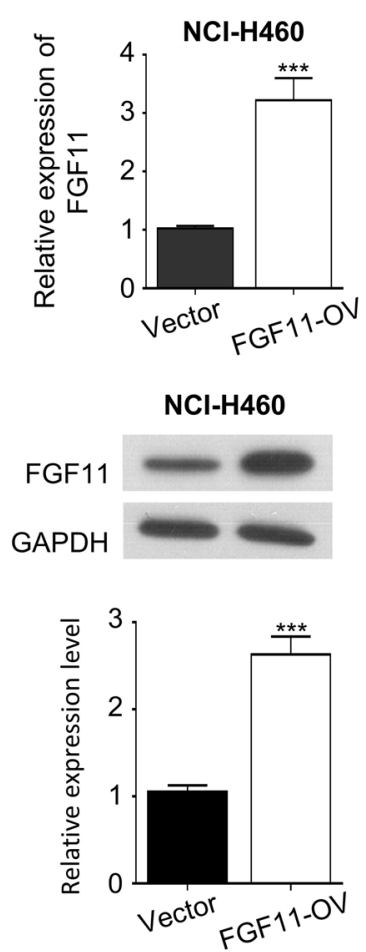

C
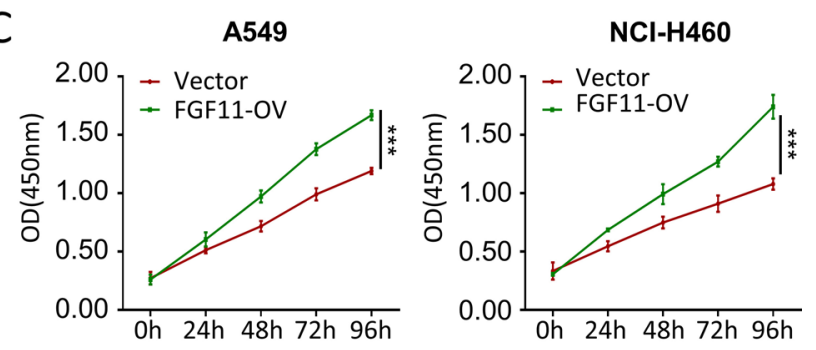

D
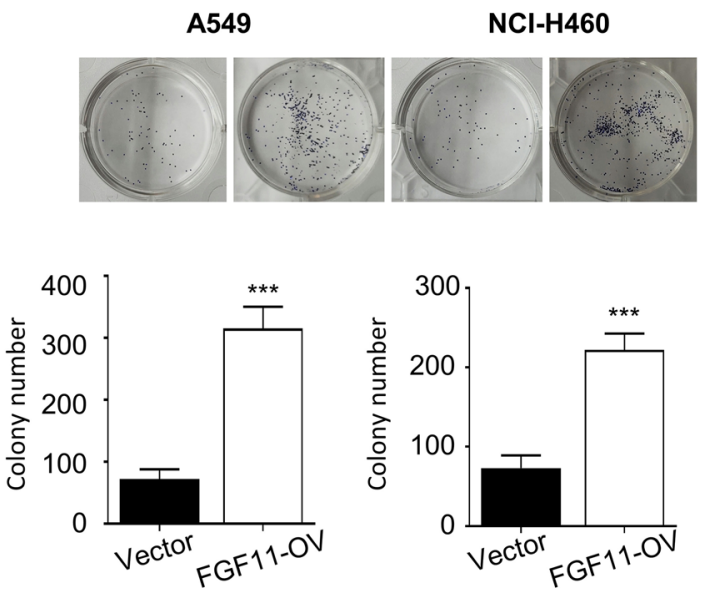

Fig. 3 Overexpression of FGF11 promotes NSCLC cell proliferation. Cells were infected with lentivirus carrying FGF11 cDNA clone or empty vector (vector). A qPCR analysis of FGF11 mRNA expression in A549 and NCl-H460 cells after lentiviral infection. B Western blot analysis of FGF11 protein levels in A549 and NCl-H460 cells. C The proliferation of A549 and NCl-H460 cells was detected by CCK-8 assay. D Colony formation assay of A549 and $\mathrm{NCl}-\mathrm{H} 460$ cells after lentiviral infection. Data are the summary of the measurements of 3 independent experiments (mean \pm standard deviation). ${ }^{*} \mathrm{P}<0.05,{ }^{* *} \mathrm{P}<0.01,{ }^{* * *} \mathrm{P}<0.005 \mathrm{vs}$. NC or empty vector-infected cells

\section{FGF11 promotes NSCLC cell migration in vitro}

We next aimed to evaluate the effect of FGF11 silencing and overexpression on the migration of NSCLC cells. Wound healing assay and transwell assay were performed to assess cell migration ability. We found that the migratory capacities of A549 and NCI-H460 cells were significantly impaired by silencing FGF11 in wound healing assay (Fig. 4A), and transwell assay (Fig. 4B). In contrast, overexpression of FGF11 remarkably enhanced the cell migration in A549 and NCI-H460 cells (Fig. 4C and D). Therefore, the migratory capacity of NSCLC is inversely correlated with FGF11 level.

\section{FGF11 is negatively regulated by miR-525-5p in NSCLC cells}

To explore the potential miRNAs regulating FGF11 expression, we first performed target scan of FGF11 sequence using three publicly available miRNA databases (miRDB, Starbase and Targetscan) (Fig. 5A). We found 4 predicted miRNAs (miR-326, miR-330-5p, miR-525-5p, miR-520a-5p) shared by three databases. We introduced miRNA mimics of the 4 microRNAs into A549 and NCI-H460 cells and analyzed FGF11 expression by qPCR. We found that miR-525-5p mimic could significantly reduce the mRNA level of FGF11 (Fig. 5B). We then cloned the wild type binding site in FGF11 3'UTR and the mutated site into a luciferase reporter and performed dual luciferase reporter assay in the presence of control or miR-525-5p mimic. Our results showed that co-transfection of miR-525-5p mimic reduced the relative luciferase activity of WT FGF11 3'UTR but had no detectable effect on the mutated FGF11 3'UTR (Fig. 5C). Meanwhile, western blot analysis also confirmed that miR-525-5p mimic could significantly reduce the protein level of FGF11 in A549 and NCI-H460 cells (Fig. 5D). Furthermore, qPCR was performed to detect the expression of miR-525-5p in NSCLC samples. The result showed that miR-525-5p expression level was significantly lower in NSCLC tumor tissues when compared to normal tissues (Fig. 5E), and there was a negative correlation between miR-525-5p level and FGF11 level (Fig. 5F).

To further examine the function of miR-525-5p on tumor cell proliferation, CCK-8 assay was performed in the presence of absence of miR-525-5p mimic. We found that miR-525-5p mimic could significantly inhibit cell 

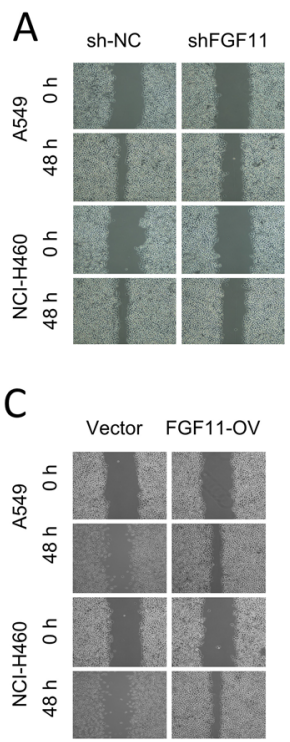

B
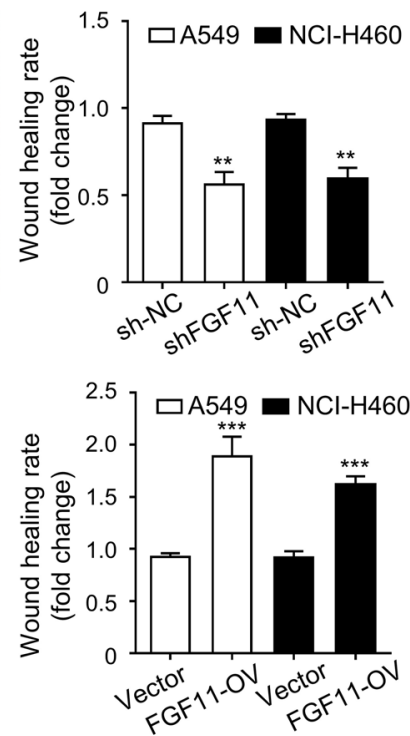

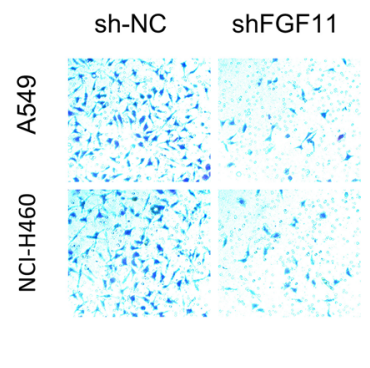

D

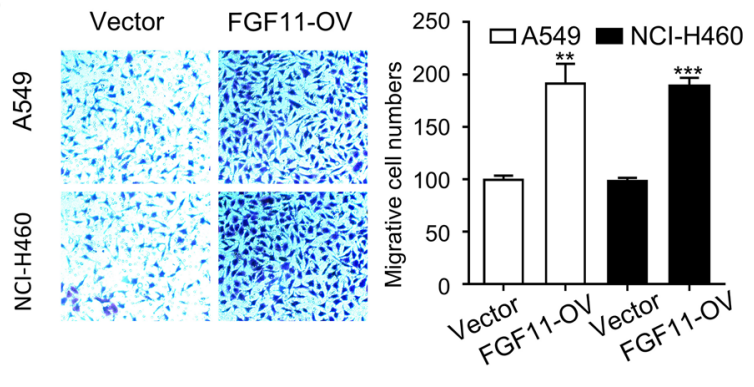

Fig. 4 FGF11 promotes NSCLC cell migration in vitro. (A-B) Cells were infected with lentivirus carrying FGF11 shRNA (shFGF11) or control shRNA (sh-NC). A Wound healing assay analysis of the migration of A549 and NCl-H460 cells after being infected with FGF11 shRNA. B Transwell assay analysis of the migration of A549 and NCI-H460 cells after being infected with FGF11 shRNA. C, D Cells were infected with lentivirus carrying FGF11 CDNA clone or empty vector (vector). C Wound healing assay analysis of the migration of A549 and NCl-H460 cells after FGF11 overexpression. D Transwell assay analysis of the migration of A549 and NCl-H460 cells after FGF11 overexpression. The results are represented as mean \pm standard derivation. Data are summary of 3 independent experiments. ${ }^{*} p<0.05,{ }^{* *} p<0.01,{ }^{* *} p<0.05{ }^{*} P<0.05,{ }^{* *} P<0.01,{ }^{* * *} P<0.005$ vs. sh-NC or empty vector-infected cells

proliferation, whereas overexpression of FGF11 partially rescued cell proliferation in A549 and NCI-H460 cells (Fig. 5G). Colony formation assay also showed the similar result (Fig. $5 \mathrm{H}$ ). Interestingly, cell migration and invasion ability were also largely suppressed by miR-525-5p mimic (Fig. 5I and J), which could also be rescued by FGF11 overexpression. Together, the above results suggest that miR-525-5p targets FGF11 in the 3' UTR and negatively control FGF11 expression and NSCLC oncogenesis.

\section{Hypoxia signaling pathway is involved} in FGF11-dependent oncogenic function

Hypoxia pathway is dysregulated in many cancers, and we next attempted to investigate whether the oncogenic function of FGF11 is related to hypoxia signaling pathway. Gene set enrichment analysis (GSEA) using GSE81089 NSCLC RNA-seq data showed that FGF11 expression was positively enriched with genes in hypoxia signaling pathway (Fig. 6A, NES $=1.85$, $\mathrm{P}<0.001$, FDR $\mathrm{q}=0.125<0.25)$. In addition, hypoxia induced factors-1 alpha (HIF-1 $\alpha$ ) plays a key role in hypoxia signaling pathways which is dysregulated lung cancer [23-26], we next explored whether FGF11 level regulates the expression of HIF- $1 \alpha$ in A549 and NCIH460 cells. Interestingly, the knockdown of FGF11 significantly reduced both the mRNA and protein level of HIF- $1 \alpha$ (Fig. $6 \mathrm{~B}$ and C). In contrast, the relative mRNA and protein level of HIF-1 $\alpha$ was increased by FGF11 overexpression in A549 and NCI-H460 cells (Fig. 6D and E).

\footnotetext{
(See figure on next page.)

Fig. 5 miR-525-5p downregulates FGF11 expression and inhibits NSCLC cell proliferation and migration. A Bioinformatic search of miRNAs targeting FGF11 using miRDB, Starbase and Targetscan databases. B qPCR analysis of FGF11 mRNA expression in A549 and NCl-H460 cells in the presence of microRNA mimics. c Dual-luciferase reporter assay of the effect of miR-525-5p mimic on FGF11 3'UTR. D western blot detected the expression of FGF11 after transfected with miR-525-5p. E qPCR analysis of the FGF11 mRNA level in NSCLC tumor tissues and normal tissues. F The correlation of miR-525-5p level and FGF11 level was examined by Pearson's correlation analysis. G CCK-8 proliferation assay of A549 and NCI-H460 cells transfected with miR-525-5p and/or FGF11. H Colony formation assay in A549 and NCI-H460 cells transfected with miR-525-5p and/or FGF11. I Wound healing assay in A549 and NCl-H460 cells transfected with miR-525-5p and/or FGF11. J Transwell migration assay in A549 and NCI-H460 cells transfected with miR-525-5p and/or FGF11. The results are represented as mean \pm standard derivation. Data are summary of 3 independent experiments. ${ }^{*} \mathrm{P}<0.05,{ }^{* *} \mathrm{P}<0.01,{ }^{* * *} \mathrm{P}<0.005$ vs. sh-NC or empty vector-transfected cells
} 


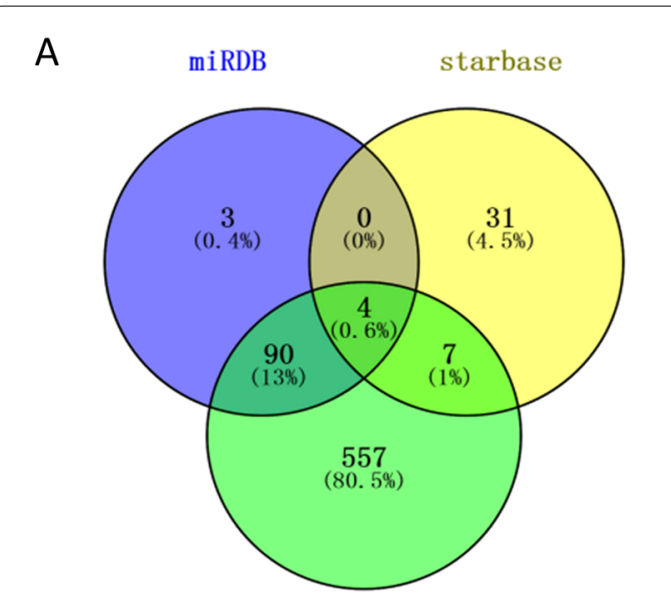

Targetscan
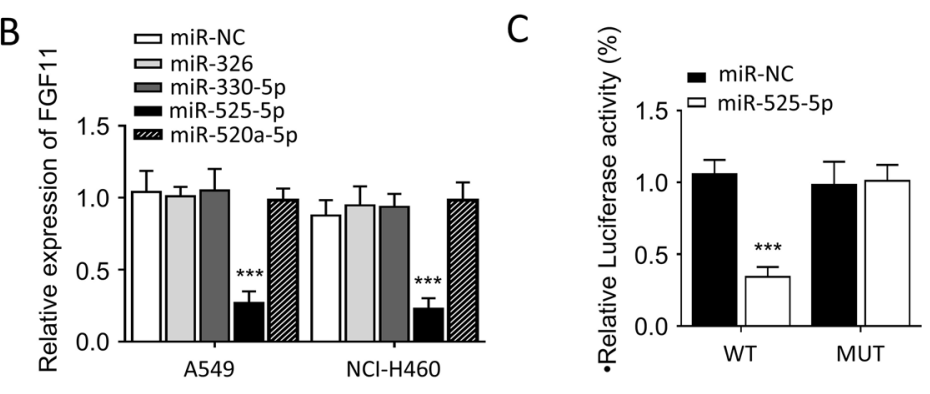

D

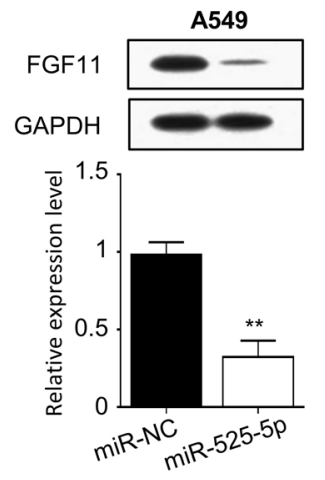

$\mathrm{F}$

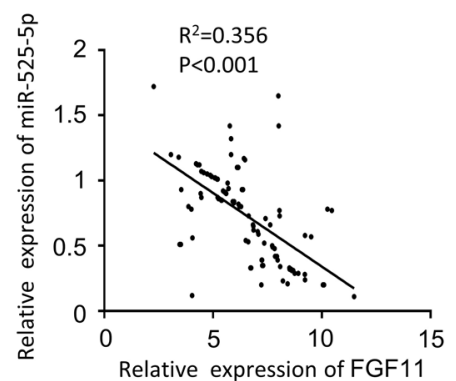

$\mathrm{H}$

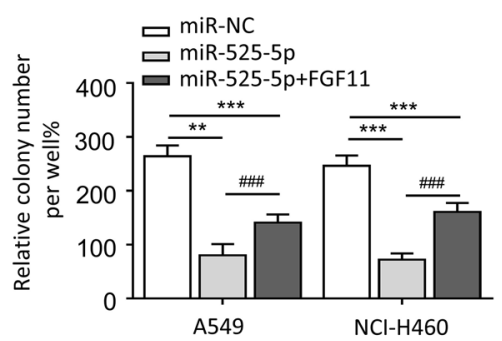

FGF11 3'UTR-WT 5'-UAUUACGGAUGAGUUCUGGAA-3'

hsa-miR-525-5p 3'-UCUUUCACGUAGGGAGACCUC-5'

FGF11 3'UTR-MUT 5'-UAUUACGGAUGAGAGCAAGCA-3'
G
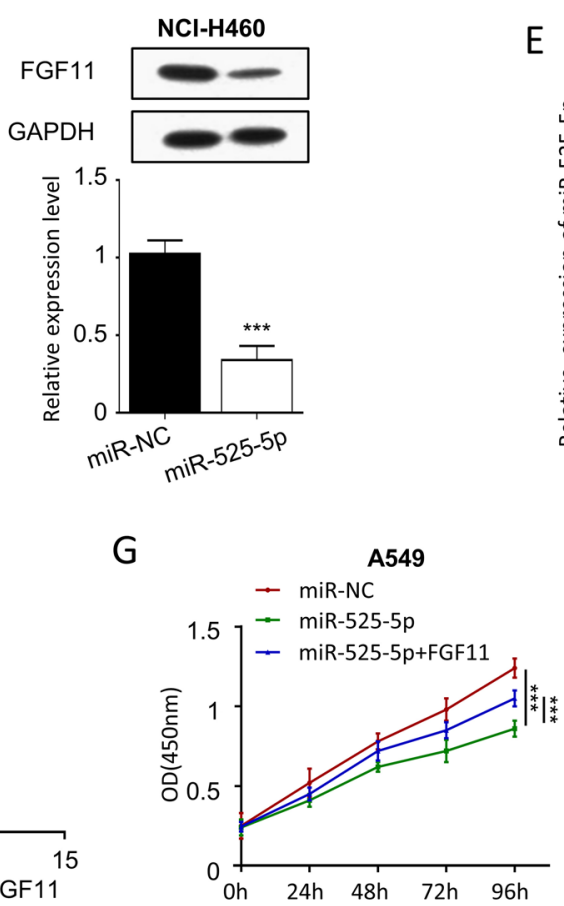

I

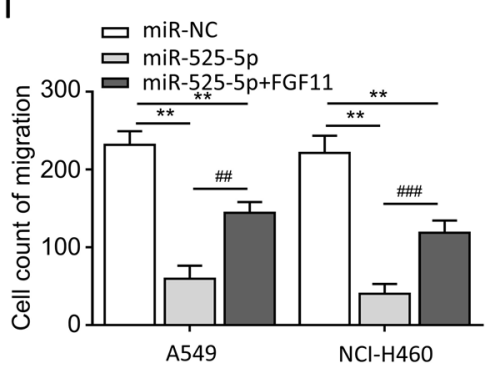

E

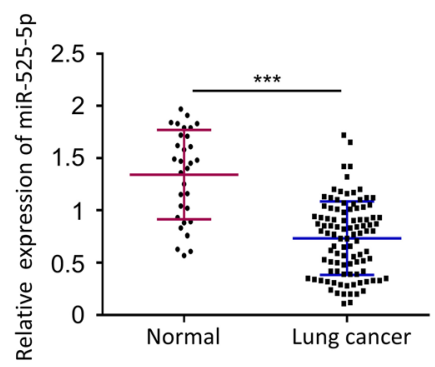

NCI-H460

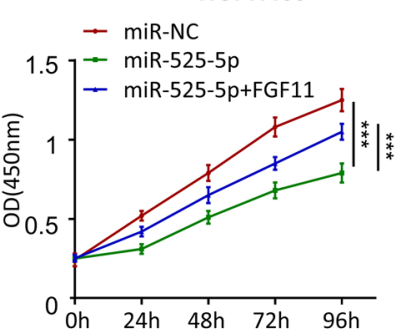

J

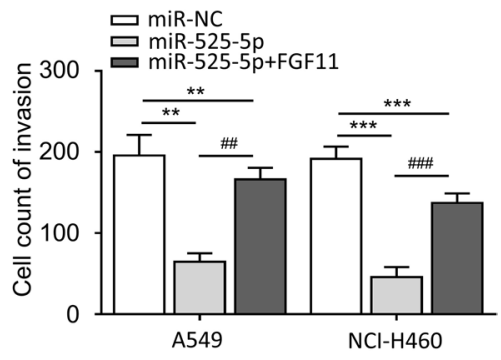

Fig. 5 (See legend on previous page.) 
(See figure on next page.)

Fig. 6 FGF11 regulates HIF-1 a expression for NSCLC progression. A Gene Set Enrichment Analysis (GSEA) of the hypoxia signaling pathway genes in FGF11 positive and negative samples usingGSE81089 RNA-seq data. B qPCR analysis of HIF-1a mRNA expression after FGF11 knockdown in A549 and NCI-H460 cells. C Western blot analysis of HIF-1a protein level after FGF11 knockdown in A549 and NCI-H460 cells. D qPCR analysis of HIF-1a mRNA expression in A549 and NCl-H460 cells with FGF11 overexpression. E Western blot analysis of HIF-1a protein level in A549 and NCI-H460 cells with FGF11 overexpression. F qPCR quantification of HIF-1 a mRNA level after siRNA transfection. G CCK-8 proliferation assay in A549 and $\mathrm{NCl}-\mathrm{H} 460$ cells transfected with FGF11 and/or HIF-1 a siRNA. H Colony formation assay in A549 and NCI-H460 cells transfected with FGF11 and/or HIF-1 a siRNA. I wound healing assay in A549 and NCI-H460 cells transfected with FGF11 and/or HIF-1a siRNA. J Transwell migration assay in A549 and $\mathrm{NCl}-\mathrm{H} 460$ cells transfected with FGF11 and/or HIF-1a siRNA. The results are represented as mean \pm standard derivation. Data are summary of 3 independent experiments. ${ }^{*} \mathrm{P}<0.05,{ }^{* *} \mathrm{P}<0.01,{ }^{* * *} \mathrm{P}<0.005 \mathrm{vs}$. sh-NC or empty vector-transfected cells

In addition, to functionally validate the involvement of HIF- $1 \alpha$ in cell proliferation, migration and invasion in A549 and NCI-H460 cells, we performed cell proliferation assay and migration assay in the presence of absence of HIF- $1 \alpha$ siRNA. qPCR analysis confirmed the downregulation of HIF- $1 \alpha$ mRNA after siRNA treatment (Fig. 6F). Our results showed that overexpression of FGF11 could significantly promote A549 and NCI-H460 cells proliferation, but HIF- $1 \alpha$ siRNA inhibited the effect of FGF11 overexpression on proliferation (Fig. 6G). Meanwhile, colony formation assay also showed the similar result (Fig. 6H). Overexpression of FGF11 enhanced cell migration in A549 and NCI-H460 cells, and HIF- $1 \alpha$ siRNA significantly impaired the effect of FGF11 (Fig. 6I and J). These results indicate that the oncogenic role of FGF11 is dependent on the upregulation of HIF- $1 \alpha$.

\section{Knockdown of FGF11 inhibits NSCLC tumor growth in vivo}

Lastly, we explored the role of FGF11 in NSCLC tumorigenesis using xenograft mouse model. In consistence with the in vitro findings, knockdown of FGF11 resulted in a significantly impaired tumorigenesis of A549 and NCI-H460 cells when injected into the nude mice (Fig. 7A and B). The IHC analysis of Ki67 (cell proliferation marker) in the tumor samples showed that knockdown of FGF11 significantly reduced the percentage of cells expressing $\mathrm{Ki}-67$ (Fig. 7C). In contrast, the overexpression of FGF11 not only promoted the tumor growth rate (Fig. 7D and E), but also increased the percentage of cells expressing Ki-67 in the tumor tissues (Fig. 7F). Collectively, these data support an oncogenic role of FGF11 in tumorigenesis of NSCLC cells.

\section{Discussion}

In recent years, lung cancer has been considered as the most prevalent cancer with highest mortality in Chinese population. Non-small cell lung cancer (NSCLC) accounts for approximately $80 \%$ of lung cancer cases, which is characterized as a heterogeneous and multifactorial disease with various genetic mutations. Targeting EGFR (epidermal growth factor receptor) and VEGF (vascular endothelial growth factor) improves the prognosis in NSCLC patients [27-30]. In addition, fibroblast growth factor (FGF) ligands and receptors have been widely implicated in a variety of tumors including lung cancer [31]. For example, FGF5 plays important roles in cell growth and invasion of human NSCLC cells [9]. Analysis of published transcriptomic data revealed that FGF11 was upregulated in NSCLC tissues, but its molecular mechanisms remain elusive.

In this study, we first confirmed the upregulation of FGF11 in both NSCLC tumor tissues and NSCLC cell lines, suggesting an oncogenic effect of FGF11. In vitro gain- and loss-of-function experiments further validated an indispensable role of FGF11 in proliferation, migration and invasion of NSCLC cells. FGF11 also prevents apoptosis in NSCLC cell lines. Besides, the oncogenic activity of FGF11 was verified in the xenograft model using NSCLC cells with stable FGF11 knockdown.

MiR-525-5p has been implicated in multiple cancers, including Glioma [32], Cervical Cancer [33]. The role of miR-525-5p in lung cancer and other human cancers remains to be investigated. Through the microRNA target scan of FGF11 and experimental validation, we found that miR-525-5p negatively regulates FGF11 expression in NSCLC cells, suggesting a tumor-suppressor role of miR-525-5p in the proliferation and migration of NSCLC cells.

Hypoxia signaling pathway has been widely dysregulated in cancer progression, [34, 35]. Previous study highlighted the role of HIF- $1 \alpha$ as a key transcriptional regulator in hypoxia signaling pathway [36]. Our results further revealed that FGF11 expression was closely correlated with hypoxia signaling pathway, and validated that FGF11 positively regulated HIF- $1 \alpha$ expression. HIF- $1 \alpha$ acts as an indispensable signaling transduction mediator by regulating key hypoxia-response genes to support tumorigenesis [37-40]. Consistently, we showed that the knockdown of HIF-1 $\alpha$ diminished the oncogenic function of FGF11 in NSCLC cells. We therefore propose that FGF11 might function as an oncogene to upregulate HIF- $1 \alpha$ and increase hypoxia signaling pathway to sustain the progression of NSCLC. 
A

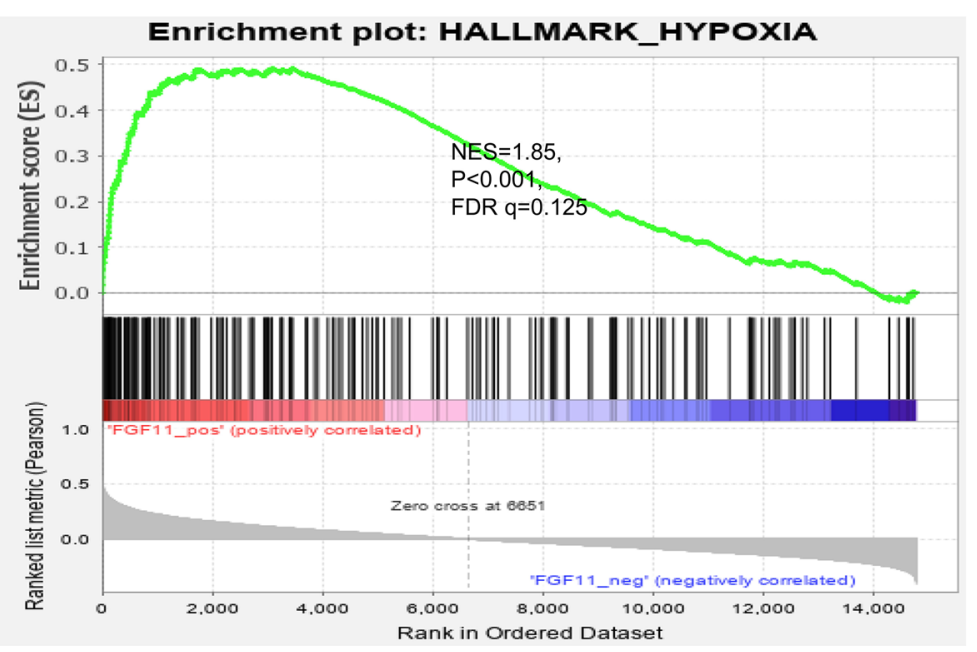

C

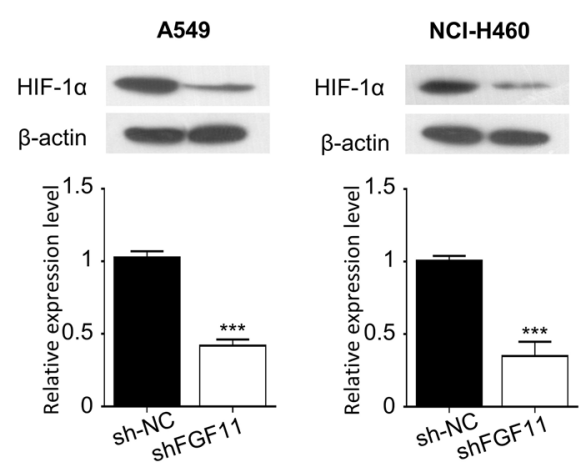

E

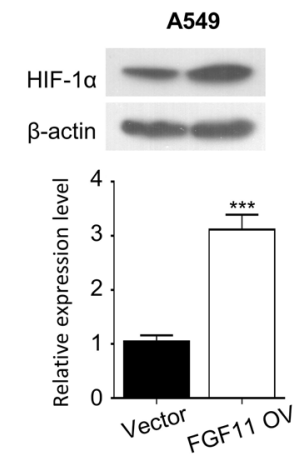

$\mathrm{H}$

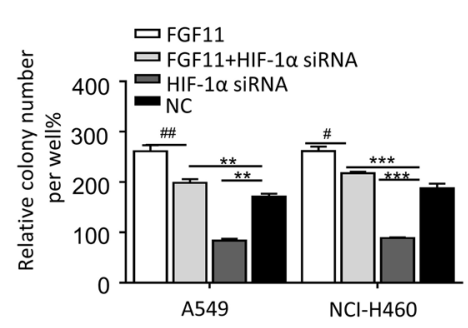

NCI-H460
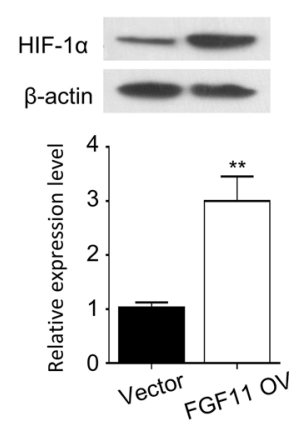

I

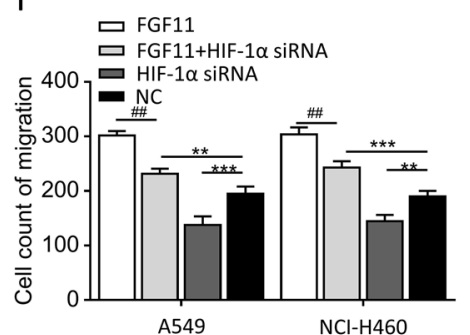

G
B
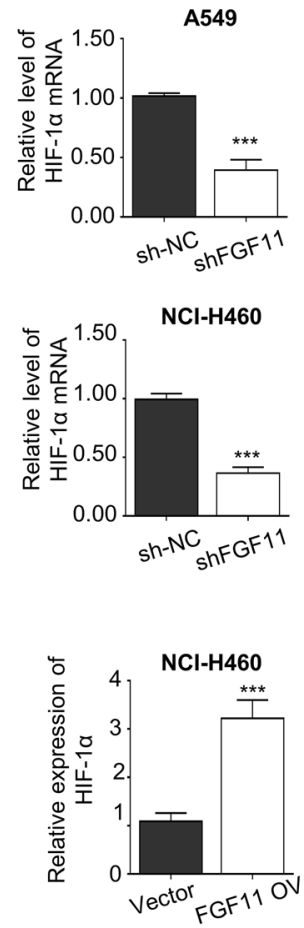

F
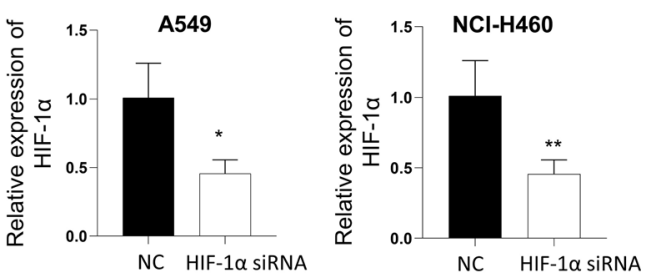<smiles>[13CH3][13CH3]</smiles>

- FGF11+HIF-1 $\alpha$ siRNA

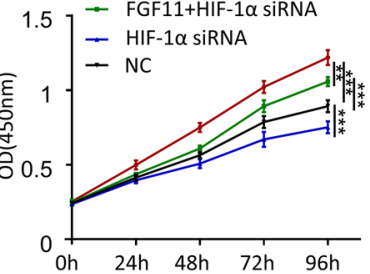

- FGF11

$1.5+\rightarrow$ FGF11+HIF-1 $\alpha$ siRNA

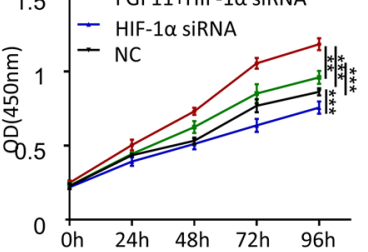

J

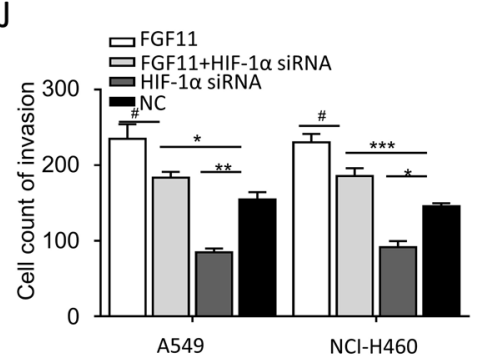

Fig. 6 (See legend on previous page.) 

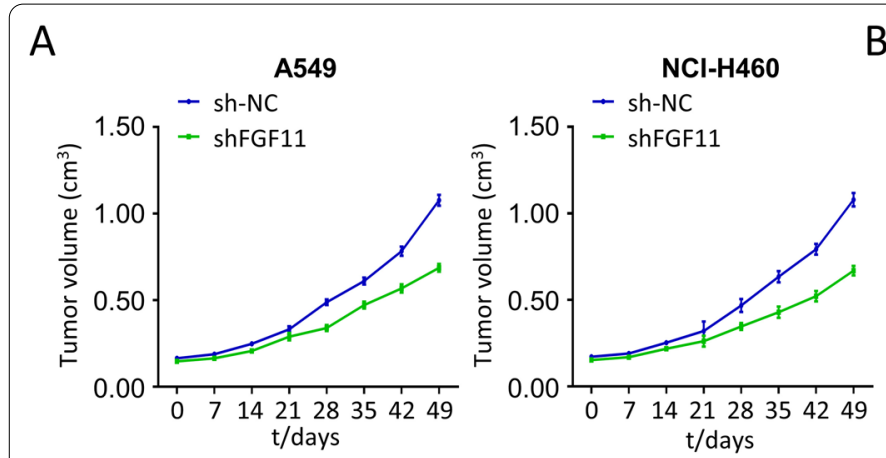

B

A549

$\mathrm{NCl}-\mathrm{H} 460$

C
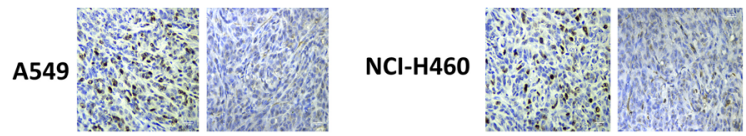

D
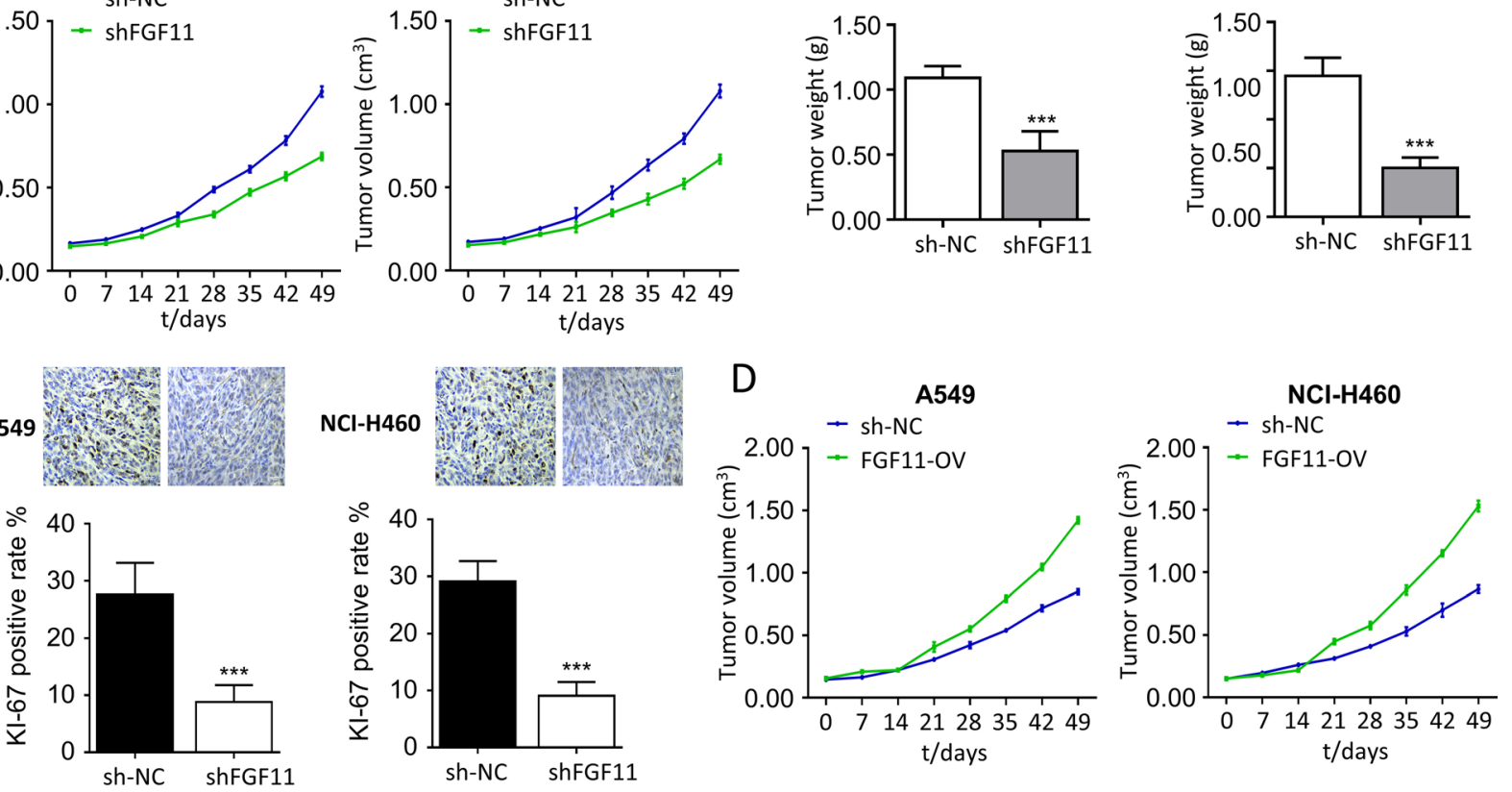

E

A549

$\mathrm{NCl}-\mathrm{H} 460$
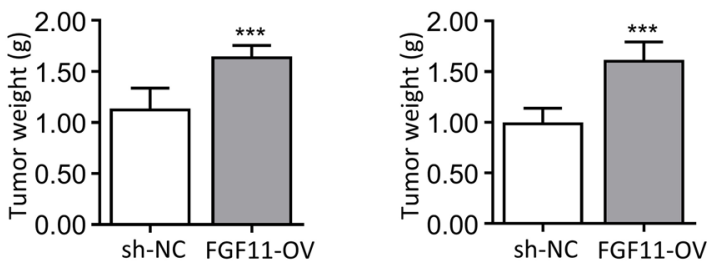

F
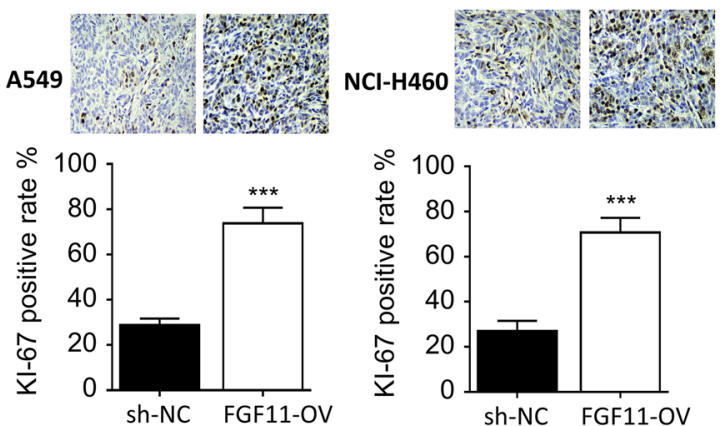

Fig. 7 FGF11 knockdown inhibits NSCLC tumorigenesis in vivo. A549 and NCI-H460 cells were infected with FGF11 shRNA and FGF11 overexpression lentivirus. Cells were inoculated into balb/c nude mice ( $n=5$ mice/group). $\mathbf{A}$ The tumor growth curve in FGF11 shRNA and control group. B Tumor weight quantification in FGF11 shRNA and control group. C Immunohistochemistry (IHC) analysis of Ki-67 expression in FGF11 shRNA and control group. D The tumor growth curve in FGF11 overexpression and control group. E Tumor weight quantification in FGF11 overexpression and control group. $\mathbf{F}$ Immunohistochemistry $(\mathrm{IHC})$ analysis of Ki-67 expression in FGF11 overexpression and control group. The results are represented as mean \pm standard derivation. ${ }^{*} \mathrm{P}<0.05,{ }^{* *} \mathrm{P}<0.01,{ }^{* * *} \mathrm{P}<0.005$ vs. control group

In conclusion, our study reveals a novel oncogenic role of FGF11 in NSCLC, which may contribute to the tumorigenesis at least partially through modulating hypoxia signaling pathway. Our findings provide evidence that targeting FGF11 can be a beneficial therapeutic strategy for the treatment of NSCLC patients with elevated FGF11 expression.

\section{Abbreviations}

FGF: Fibroblast growth factor; NSCLC: Non-small cell lung cancer; HIF: Hypoxia inducible factor; miRNAs: MicroRNAs; CCK-8: Cell counting kit-8.

\section{Supplementary Information}

The online version contains supplementary material available at https://doi. org/10.1186/s12967-021-03018-7.

Additional file 1: Table S1. Clinical pathological features of the selected 100 non-small cell lung cancer patients.

\section{Acknowledgements}

The authors declare that there are no competing interests.

\section{Authors' contributions}

XW and ML: experiment studies, clinical studies, Data collection and Manuscript writing; YD, SK, FL and YW: interpreted the patient data, Data collection 
and Manuscript writing; SZ: Project design, data analysis, manuscript editing All authors read and approved the final manuscript.

\section{Funding}

This work was supported by Natural Science Fundation of Hubei Province (2020CFB740), National Natural Science Foundation of China (82001785) and Natural Science Foundation of Hubei Province, China (2019CFB437).

\section{Declarations}

\section{Ethics approval and informed consents}

The use of human tissues was approved by the Ethics Committee of Tongji Hospital, all patients and/or their relatives signed the informed consents. All animal experimental procedures were approved by the Ethics Committee of Tongji Hospital.

\section{Author details}

'Department of Thoracic Surgery, Ersity of Science and Technology, Tongji Hospital, Tongji Medical Collage of Huazhong Univ, 430030, Wuhan, Hubei, China. ${ }^{2}$ Department of Thoracic Surgery, Zhongshan Hospital, Xiamen University, Xiamen 361004, Fujian, China. ${ }^{3}$ Department of Nuclear Medicine, Dongzhimen Hospital of Beijing University of Chinese Medicine, Beijing, China. ${ }^{4}$ Department of Emergency Medicine, Tongji Hospital, Tongji Medical College of Huazhong University of Science and Technology, 430030, Wuhan, Hubei, China. ${ }^{5}$ Department of Radiology, Tongji Hospital, Tongji Medical Collage of Huazhong University of Science and Technology, 430030, Wuhan, Hubei, China.

Received: 11 April 2021 Accepted: 31 July 2021

Published online: 17 August 2021

\section{References}

1. Bray F, et al. Global cancer statistics 2018: GLOBOCAN estimates of incidence and mortality worldwide for 36 cancers in 185 countries. CA Cancer I Clin. 2018;68(6):394-424.

2. Kim D, et al. Lung cancer staging and associated genetic and epigenetic events. Mol Cells. 2020;43(1):1-9.

3. Onoi K, et al. Immune checkpoint inhibitors for lung cancer treatment: a review. J Clin Med. 2020;9:5.

4. Iams WT, Porter J, Horn L. Immunotherapeutic approaches for small-cell lung cancer. Nat Rev Clin Oncol. 2020;17(5):300-12.

5. Jeong Y, et al. Role of KEAP1/NFE2L2 mutations in the chemotherapeutic response of patients with non-small cell lung cancer. Clin Cancer Res. 2020:26(1):274-81.

6. Ban MJ, et al. Fibroblast growth factor receptor 3-mediated reactivation of ERK signaling promotes head and neck squamous cancer cell insensitivity to MEK inhibition. Cancer Sci. 2018;109(12):3816-25.

7. Garje R, et al. Fibroblast Growth Factor Receptor (FGFR) Inhibitors in Urothelial Cancer. Oncologist. 2020;25(11):e1711.

8. He L, et al. Downregulation of basic fibroblast growth factor increases cisplatin sensitivity in A549 non-small cell lung cancer cells. J Cancer Res Ther 2018;14(7):1519-24.

9. Zhou Y, et al. Downregulation of fibroblast growth factor 5 inhibits cell growth and invasion of human nonsmall-cell lung cancer cells. J Cell Biochem. 2018;120(5):8238-46.

10. Lee KW, et al. FGF11 induced by hypoxia interacts with HIF-1alpha and enhances its stability. FEBS Lett. 2017;591(2):348-57.

11. Ornitz DM, Itoh N. The Fibroblast Growth Factor signaling pathway. Wiley Interdiscip Rev Dev Biol. 2015;4(3):215-66.

12. Gebert LFR, MacRae IJ. Regulation of microRNA function in animals. Nat Rev Mol Cell Biol. 2019;20(1):21-37.

13. O'Brien J, et al. Overview of MicroRNA biogenesis, mechanisms of actions, and circulation. Front Endocrinol (Lausanne). 2018;9:402.

14. Shin VY, Chu KM. MiRNA as potential biomarkers and therapeutic targets for gastric cancer. World J Gastroenterol. 2014;20(30):10432-9.

15. McGuire A, Brown JA, Kerin MJ. Metastatic breast cancer: the potential of miRNA for diagnosis and treatment monitoring. Cancer Metastasis Rev. 2015;34(1):145-55.
16. Iqbal MA, et al. MicroRNA in lung cancer: role, mechanisms, pathways and therapeutic relevance. Mol Aspects Med. 2019;70:3-20.

17. Jung $G$, et al. Epigenetics of colorectal cancer: biomarker and therapeutic potential. Nat Rev Gastroenterol Hepatol. 2020;17(2):111-30.

18. Ye SB, et al. Exosomal miR-24-3p impedes T-cell function by targeting FGF11 and serves as a potential prognostic biomarker for nasopharyngeal carcinoma. J Pathol. 2016;240(3):329-40.

19. Hu S, et al. Infiltrating T cells promote prostate cancer metastasis via modulation of FGF11->miRNA-541->androgen receptor (AR)->MMP9 signaling. Mol Oncol. 2015;9(1):44-57.

20. Knowles HJ. Hypoxia-induced fibroblast growth factor 11 stimulates osteoclast-mediated resorption of bone. CalcifTissue Int. 2017;100(4):382-91.

21. Yang J, et al. Hypoxia-induced fibroblast growth factor 11 stimulates capillary-like endothelial tube formation. Oncol Rep. 2015;34(5):2745-51.

22. Lee $\mathrm{M}$, et al. Copy number variations of chromosome 17p13.1 might be linked to high risk of lung cancer in heavy smokers. Mol Biol Rep. 2011;38(8):5211-7.

23. Ahmmed B, et al. Rg3 inhibits gemcitabine-induced lung cancer cell invasiveness through ROS-dependent, NF-kappaB- and HIF-1alpha-mediated downregulation of PTX3. J Cell Physiol. 2019;234(7):10680-97.

24. D'Ignazio L, Batie M, Rocha S. Hypoxia and Inflammation in Cancer Focus on HIF and NF-kappaB. Biomedicines. 2017;5:2.

25. Liu J, et al. PI3K/Akt/HIF-1 alpha signaling pathway mediates HPV-16 oncoprotein-induced expression of EMT-related transcription factors in nonsmall cell lung cancer cells. J Cancer. 2018;9(19):3456-66.

26. Salem $A$, et al. Targeting hypoxia to improve non-small cell lung cancer outcome. J Natl Cancer Inst. 2018;110:1.

27. Chen $X$, et al. Cutting edge: expression of TNFR2 defines a maximally suppressive subset of mouse CD4+CD25+FoxP3+T regulatory cells: applicability to tumor-infiltrating T regulatory cells. J Immunol. 2008;180(10):6467-71.

28. Chen Y, Mathy NW, Lu H. The role of VEGF in the diagnosis and treatment of malignant pleural effusion in patients with nonsmall cell lung cancer (Review). Mol Med Rep. 2018;17(6):8019-30.

29. Hsu PC, et al. Epidermal Growth Factor Receptor (EGFR) Pathway, YesAssociated Protein (YAP) and the Regulation of Programmed Death-Ligand 1 (PD-L1) in Non-Small Cell Lung Cancer (NSCLC). Int J Mol Sci. 2019;20:15.

30. Masuda C, et al. Bevacizumab counteracts VEGF-dependent resistance to erlotinib in an EGFR-mutated NSCLC xenograft model. Int J Oncol. 2017;51(2):425-34.

31. Semrad TJ, Mack PC. Fibroblast growth factor signaling in non-small-cell lung cancer. Clin Lung Cancer. 2012;13(2):90-5.

32. Xie P, et al. miR-525-5p modulates proliferation and epithelial-mesenchymal transition of glioma by targeting stat-1. Onco Targets Ther. 2020;13:9957-66.

33. Chen M, Liu LX. MiR-525-5p repressed metastasis and anoikis resistance in cervical cancer via blocking UBE2C/ZEB1/2 signal axis. Dig Dis Sci. 2020;65(8):2442-51.

34. Marchiq I, Pouyssegur J. Hypoxia, cancer metabolism and the therapeutic benefit of targeting lactate/ $\mathrm{H}(+)$ symporters. J Mol Med (Berl). 2016;94(2):155-71.

35. Mennerich D, Kubaichuk K, Kietzmann T. DUBs, hypoxia, and cancer. Trends Cancer. 2019;5(10):632-53.

36. Masoud GN, Li W. HIF-1alpha pathway: role, regulation and intervention for cancer therapy. Acta Pharm Sin B. 2015;5(5):378-89.

37. Liu J, et al. Parkin targets HIF-1alpha for ubiquitination and degradation to inhibit breast tumor progression. Nat Commun. 2017;8(1):1823.

38. Palazon A, et al. An HIF-1alphaNEGF-A Axis in Cytotoxic T cells regulates tumor progression. Cancer Cell. 2017;32(5):669-83.

39. Yang N, et al. Propofol suppresses LPS-induced nuclear accumulation of HIF1 lalpha and tumor aggressiveness in non-small cell lung cancer. Oncol Rep. 2017;37(5):2611-9.

40. Zhang $\mathrm{H}$, et al. Hypoxia-inducible factor-1alpha (HIF-1 alpha) promotes hypoxia-induced invasion and metastasis in ovarian cancer by targeting matrix metallopeptidase 13 (MMP13). Med Sci Monit. 2019;25:7202-8.

\section{Publisher's Note}

Springer Nature remains neutral with regard to jurisdictional claims in published maps and institutional affiliations. 